\title{
Convergence of immunotherapy, radiotherapy and prostate cancer: challenges and opportunities
}

\author{
Michael Green ${ }^{1}$, Felix Y Feng ${ }^{2}$, Rohit Mehra ${ }^{3} \&$ Daniel E Spratt ${ }^{*}, 1$ \\ ${ }^{1}$ Department of Radiation Oncology, University of Michigan, Ann Arbor, MI, USA \\ ${ }^{2}$ Department of Radiation Oncology, University of California San Francisco, San Francisco, CA, USA \\ ${ }^{3}$ Department of Pathology, University of Michigan, Ann Arbor, MI, USA \\ *Author for correspondence: Tel.: +1 734647 1372; Fax: +1 734936 1900; sprattda@med.umich.edu
}

First draft submitted: 11 May 2017; Accepted for publication: 6 June 2017; Published online: 3 August 2017

Keyw ords: immunotherapy • prostate cancer • radiotherapy

Immunotherapy has led to unprecedented and durable response rates in multiple cancer types. However, similar success in prostate cancer has not been achieved, leaving patients and the prostate cancer scientific community working harder than ever to understand why and how to identify and select patients who may benefit from these powerful therapies. In parallel, the use of immunotherapy with radiotherapy has demonstrated immense promise in preclinical testing. Given the fundamental role radiotherapy has in the treatment of both primary and metastatic prostate cancer, there is strong rationale for the combination of these two treatments to improve outcomes for prostate cancer patients. Herein, we discuss the state of immunotherapy for prostate cancer and the current obstacles and opportunities available under active investigation.

Prostate cancer is the most common noncutaneous malignancy in men in the USA [1]. However, there remain 26,700 deaths from prostate cancer each year making death from prostate cancer more common than renal cell carcinoma, melanoma and all brain tumors combined each year in men. The primary methods of treatment intensification for metastatic castration-resistant prostate cancer (mCRPC) are intensification of androgen signaling inhibition or addition of cytotoxic chemotherapy. However, few CRPC patients achieve long-term durable control of their disease and ubiquitously succumb to their disease. Thus, there is an unmet clinical need for additional therapies to improve the care of patients with prostate cancer.

Immunotherapy is a true disruptive therapeutic innovation in oncology. Unprecedented response rates, and perhaps more importantly durable response rates, have been achieved in metastatic renal cell carcinoma, melanoma, non-small-cell lung cancer, bladder cancer, Merkel cell carcinoma, lymphoma, among others. However, similarly designed clinical trials in prostate cancer have not demonstrated comparable rates of efficacy leaving patients and the prostate cancer scientific community working harder than ever to understand why and how to identify and select patients who may benefit from these powerful therapies.

In parallel to the surge of positive trials with immunotherapy in other disease sites, the use of immunotherapy with radiotherapy has demonstrated immense promise in primarily preclinical testing. Given the fundamental role radiotherapy has in the treatment of both primary and metastatic prostate cancer for curative intent or palliative benefit, there is strong rationale for the combination of these two treatments to improve outcomes for our patients. Herein, we discuss the state of immunotherapy for prostate cancer and the current obstacles and opportunities available under active investigation.

\section{History of immunotherapy in prostate cancer}

Prostate cancer pioneered early immunostimulatory efforts. Sipuleucel-T, which consists of ex vivo dendritic cell stimulation with granulocyte-macrophage colony-stimulating factor and the conserved tumor antigen prostatic alkaline phosphatase, was the first US FDA approved cellular immunotherapy in 2010 on the basis of the IMPACT trial [2]. This trial randomized men with minimally symptomatic hormone-resistant metastatic prostate cancer to 
placebo or sipuleucel-T. The trial met its primary end point of improved overall survival with a median benefit of 4.1 months, but surprisingly did not induce tumor regression or prevent tumor progression.

More recently, immune checkpoint blockade (ICB) has emerged as a novel therapy capable of producing durable widespread responses across a broad spectrum of malignancies [3,4]. The current FDA-approved agents target CTLA-4 (ipilumimab), or the ligand-receptor conjugate of PD-1 (e.g., nivolumab, pembrolizumab) and PD-L1 (e.g., atezolizumab) to improve adaptive and innate antitumoral immune responses [5]. Unfortunately, randomized control trials examining the role of ipilumimab in the treatment of mCRPC failed to meet their primary end point of improvement in overall survival [6].

Initial Phase I/II studies of nivolumab for a variety of metastatic solid tumors also failed to show a response rate for $\mathrm{mCRPC}$ [7]. Preliminary Phase II data have suggested that enzalutamide-resistant prostate cancer patients may have as high as a $30 \%$ response rate to pembrolizumab monotherapy, comparable to the rate seen in many other malignancies [8]. While encouraging, this trial has reported on only ten patients to date, and it is unclear if sequencing of therapies or improved patient selection led to the improved response rate for the use of ICB in prostate cancer. Given that there is little to no PD-L1 expression in primary prostate cancer, it is plausible given the increased mutational burden and chromosomal instability of mCRPC that immunotherapy may be more efficacious in this setting. The STARVE-PC trial is examining combination therapy with anti-CTLA4 and anti-PD-1 antibodies in a subset of mCRPC-expressing AR-V7 therapies (NCT02601014), given that this combination has shown to be more efficacious in melanoma therapy as compared with monotherapy.

\section{Biological basis for immunotherapy in prostate cancer}

The limited efficacy of immune checkpoint therapy has led to renewed immunological evaluation of prostate cancer. It has been suggested that the low mutational burden of prostate cancer limits the immunogenicity of the tumor [9]. Prostate cancer does, however have many public tissue-associated antigens, including PSA, PMSA, prostatic alkaline phosphatase (the target of sipuleucel-T) and NY-ESO, and there are a variety of Phase I studies examining the potential to target these antigens to elicit an immune response [10]. It is increasingly recognized that tumors undergo a process of adaptive immunoediting which allows the selection and outgrowth of subclones that can grow in an immunocompetent host [11].

Prostate cancer has a long natural history, and autopsy series has shown that even young adult men can have low-grade prostate cancer. This suggests that prostate cancer has a long phase of immune equilibrium during which the immune system can keep the cancer in check [12]. Consistent with this, patients who are placed on chronic immunosuppressive regimens or who develop viral immunosuppression develop a higher rate of solid tumors, including prostate cancer [13]. Further, while tumoral PD-L1 and T-cell PD-1 expression does not ubiquitously serve as a predictive biomarker in response to ICB, multiple studies have shown that prostate cancer expresses PD-L1 and prostate-infiltrating T cells express PD-1 [14,15].

Recently, it has been reported that in enzalutamide-resistant PTEN deleted murine prostate cancer models, the limited efficacy of ICB stems from an immunosuppressive tumor milieu composed of myeloid-derived suppressor cells. Combination of myeloid-derived suppressor cell targeted agents with ICB showed improved tumor elimination, forming the basis for future clinical trials [16]. Finally, other reports have highlighted that ipilumimab and androgen deprivation therapy (ADT) of prostate cancer patients may induce additional immunoinhibitory compensatory mechanisms, including V-domain immunoglobulin (Ig)-containing suppressor of T-cell activation (VISTA) expression [17]. Thus there is a strong biological basis for immunotherapy in the treatment of prostate cancer and several promising leads to potentiate current ICB therapy have been identified.

\section{Radiotherapy \& immunotherapy for prostate cancer}

Radiotherapy is a noninvasive prostate cancer treatment modality widely utilized both for the definitive management and palliation of painful bony metastases [18]. Radiotherapy induces complex DNA double-strand breaks in tumors, which if not repaired, lead mitotic catastrophe and tumor cell death. On a cellular level, radiotherapy-induced cell death releases danger-associated molecular patterns, such as HMGB1, which induces dendritic cell activation through TLR4 signaling [19]. Further, the DNA damage induced by radiation releases cytoplasmic DNA which elicits Type I interferon signaling and immune activation in a cGAS-STING dependent manner [20].

Preclinically, ionizing radiation has been shown to synergize with ICB by promoting T-cell diversity, improving MHC presentation and inducing abscopal tumor rejection even in nonirradiated lesions [21,22]. Case reports have extended this paradigm to human patients [23]. Despite promising Phase I and II studies, a randomized Phase III 
trial of adjuvant ipilumimab following ionizing radiation failed to meet the primary end point of improved overall survival. However, improvements of progression-free survival were seen and there are questions of whether the correct sequence of therapies and dose of radiation were used [24,25].

Hypofractionated radiation schedules that elicit immunologic cell death are also increasingly being utilized for the treatment of definitive prostate cancer [26]. Thus there is a clinical opportunity to examine the role of immunotherapy-based treatment intensification through combination with radiotherapy. However, immunotherapy is associated with serious autoimmune side effects. Institutional studies of prostate cancer patients treated with ipilumimab highlight that CTLA- 4 treatments have significant risk of developing grade 3-4 hypopituitarism (18.2\%), dermatitis (6.8\%) and enterocolotis (15.9\%) [27]. Prostate-directed radiotherapy can also cause acute and chronic bowel toxicity [28], and thus treatment intensification will need to be balanced with the potential for increased toxicity profiles.

The interface between hormonal therapy and immunotherapy remains undefined. ADT has been suggested to function by potentiating the DNA damage induced by ionizing radiation, and by reducing the testosterone that fuels prostate cancer growth [29]. Testosterone has long been recognized to contribute to immunologic aging, and castration can lead to improved T-cell number and diversity by limiting thymic involution and contribution to peripheral T-cell homeostasis [30-32]. Murine and human studies have shown that ADT increases T-cell infiltration into the prostate and improves antitumor-associated antigen recognition, suggesting that testosterone may limit host recognition by inducing Ptpn1 and preventing Th1 differentiation [33-36]. ADT has also been shown to potentiate ipilumimab in Phase II trials of prostate cancer [37]. Thus, whether ADT can be exploited to improve immunotherapies remains to be fully assessed.

\section{Conclusion}

Despite a series of trials that have not met their primary end point to improve overall survival in prostate cancer, a tremendous amount of knowledge has been gained to potentially help explain the clinical results seen to date. There is a small subset of prostate cancers that harbor a high mutational burden and they should undergo dedicated investigation to determine if comparable response rates can be achieved to other hypermutated tumors [38]. Furthermore, given the low PD-L1 expression of prostate cancer, methods to either increase PD-L1 expression, or investigation, of new therapeutic targets may be necessary. Regardless, immunotherapy continues to hold tremendous promise even for prostate cancer and we anxiously await the results of the many ongoing clinical trials.

Financial \& competing interests disclosure

DE Spratt was supported by the Prostate Cancer Foundation Young Investigator Award (DES). He served on an advisory board for Dendreon. FY Feng served on advisory boards for M edivation/Astellas, GenomeDx, Nanostring, Celgene, Dendreon and had grant funding from Varian, Medivation/Astellas and Celgene. The authors have no other relevant affiliations or financial involvement with any organization or entity with a financial interest in or financial conflict with the subject matter or materials discussed in the manuscript apart from those disclosed.

No writing assistance was utilized in the production of this manuscript.

\section{References}

1 Siegel RL, Miller KD, Jemal A. Cancer statistics, 2016. CA Cancer J. Clin. 66(1), 7-30 (2016).

2 Kantoff PW, Higano CS, Shore ND et al. Sipuleucel-T immunotherapy for castration-resistant prostate cancer. N. Engl. J. Med. 363(5), 411-422 (2010).

3 Postow MA, Callahan MK, Wolchok JD. Immune checkpoint blockade in cancer therapy. J. Clin. Oncol. 33(17), 1974-1982 (2015).

4 Homet Moreno B, Ribas A. Anti-programmed cell death protein-1/ligand-1 therapy in different cancers. Br. J. Cancer 112(9), 1421-1427 (2015).

5 Zou W, Wolchok JD, Chen L. PD-L1 (B7-H1) and PD-1 pathway blockade for cancer therapy: mechanisms, response biomarkers, and combinations. Sci. Transl. Med. 8(328), 328rv4 (2016).

6 Beer TM, Kwon ED, Drake CG et al. Randomized, double-blind, Phase III trial of ipilimumab versus placebo in asymptomatic or minimally symptomatic patients with metastatic Chemotherapy-naive castration-resistant prostate cancer. J. Clin. Oncol. 35(1), 40-47 (2017).

7 Bracarda S, Altavilla A, Hamzaj A et al. Immunologic checkpoints blockade in renal cell, prostate, and urothelial malignancies. Semin. Oncol. 42(3), 495-505 (2015). 
8 Graff JN, Alumkal JJ, Drake CG et al. Early evidence of anti-PD-1 activity in enzalutamide-resistant prostate cancer. Oncotarget 7(33), 52810-52817 (2016).

9 Alexandrov LB, Nik-Zainal S, Wedge DC et al. Signatures of mutational processes in human cancer. Nature 500(7463), 415-421 (2013).

10 Kiessling A, Wehner R, Füssel S, Bachmann M, Wirth MP, Schmitz M. Tumor-associated antigens for specific immunotherapy of prostate cancer. Cancers (Basel) 4(1), 193-217 (2012).

11 Dunn GP, Bruce AT, Ikeda H, Old LJ, Schreiber RD. Cancer immunoediting: from immunosurveillance to tumor escape. Nat. Immunol. 3(11), 991-998 (2002).

12 Bell KJ, Del Mar C, Wright G, Dickinson J, Glasziou P. Prevalence of incidental prostate cancer: a systematic review of autopsy studies. Int. J. Cancer 137(7), 1749-1757 (2015).

13 Birkeland SA, Storm HH, Lamm LU et al. Cancer risk after renal transplantation in the Nordic countries, 1964-1986. Int. J. Cancer 60(2), 183-189 (1995).

14 Taube JM, Klein A, Brahmer JR et al. Association of PD-1, PD-1 ligands, and other features of the tumor immune microenvironment with response to anti-PD-1 therapy. Clin. Cancer. Res. 20(19), 5064-5074 (2014).

15 Sfanos KS, Bruno TC, Meeker AK, De Marzo AM, Isaacs WB, Drake CG. Human prostate-infiltrating CD8 ${ }^{+}$T lymphocytes are oligoclonal and PD-1+. Prostate 69(15), 1694-1703 (2009).

16 Lu X, Horner JW, Paul E et al. Effective combinatorial immunotherapy for castration-resistant prostate cancer. Nature 543(7647), 728-732 (2017).

17 Gao J, Ward JF, Pettaway CA et al. VISTA is an inhibitory immune checkpoint that is increased after ipilimumab therapy in patients with prostate cancer. Nat. Med. 23(5), 551-555 (2017).

18 Devita, Hellman, and Rosenberg's Cancer: Principles and Practice of Oncology Review. Wolters Kluwer Health, (2012).

19 Apetoh L, Ghiringhelli F, Tesniere A et al. Toll-like receptor 4-dependent contribution of the immune system to anticancer chemotherapy and radiotherapy. Nat. Med. 13(9), 1050-1059 (2007).

20 Deng L, Liang H, Xu M et al. STING-dependent cytosolic DNA sensing promotes radiation-induced Type I interferon-dependent antitumor immunity in immunogenic tumors. Immunity 41(5), 843-852 (2014).

21 Twyman-Saint Victor C, Rech AJ, Maity A et al. Radiation and dual checkpoint blockade activate non-redundant immune mechanisms in cancer. Nature 520(7547), 373-377 (2015).

22 Demaria S, Golden EB, Formenti SC. Role of local radiation therapy in cancer immunotherapy. JAMA Oncology 1(9), 1325 (2015).

23 Postow MA, Callahan MK, Barker CA et al. Immunologic correlates of the abscopal effect in a patient with melanoma. N. Engl. J. Med. 366(10), 925-931 (2012).

24 Kwon ED, Drake CG, Scher HI et al. Ipilimumab versus placebo after radiotherapy in patients with metastatic castration-resistant prostate cancer that had progressed after docetaxel chemotherapy (CA184-043): a multicentre, randomised, double-blind, Phase III trial. Lancet Oncol. 15(7), 700-712 (2014).

25 Slovin SF, Beer TM, Higano CS et al. Initial Phase II experience of ipilimumab (IPI) alone and in combination with radiotherapy (XRT) in patients with metastatic castration-resistant prostate cancer (mCRPC). J. Clin. Oncol. 27(15S), 5138 (2009).

26 King CR, Brooks JD, Gill H, Presti JC Jr. Long-term outcomes from a prospective trial of stereotactic body radiotherapy for low-risk prostate cancer. Int. J. Radiat. Oncol. Biol. Phys. 82(2), 877-882 (2012).

27 Gao J, He Q, Subudhi $S$ et al. Review of immune-related adverse events in prostate cancer patients treated with ipilimumab: MD Anderson experience. Oncogene 34(43), 5411-5417 (2015).

28 Spratt DE, Pei X, Yamada J, Kollmeier MA, Cox B, Zelefsky MJ. Long-term survival and toxicity in patients treated with high-dose intensity modulated radiation therapy for localized prostate cancer. Int. J. Radiat. Oncol. Biol. Phys. 85(3), 686-692 (2013).

29 Polkinghorn WR, Parker JS, Lee MX et al. Androgen receptor signaling regulates DNA repair in prostate cancers. Cancer Discov. 3(11), 1245-1253 (2013).

30 Montecino-Rodriguez E, Berent-Maoz B, Dorshkind K. Causes, consequences, and reversal of immune system aging. J. Clin. Invest. 123(3), 958-965 (2013).

31 Green MD, Snoeck HW. Novel approaches for immune reconstitution and adaptive immune modeling with human pluripotent stem cells. BMC Med. 9, 51 (2011).

32 Franceschi C, Bonafe M, Valensin S. Human immunosenescence: the prevailing of innate immunity, the failing of clonotypic immunity, and the filling of immunological space. Vaccine 18(16), 1717-1720 (2000).

33 Kissick HT, Sanda MG, Dunn LK et al. Androgens alter T-cell immunity by inhibiting T-helper 1 differentiation. Proc. Natl Acad. Sci. USA 111(27), 9887-9892 (2014).

34 Arredouani MS, Tseng-Rogenski SS, Hollenbeck BK et al. Androgen ablation augments human HLA2.1-restricted T cell responses to PSA self-antigen in transgenic mice. Prostate 70(9), 1002-1011 (2010). 
35 Roden AC, Moser MT, Tri SD et al. Augmentation of T cell levels and responses induced by androgen deprivation. J. Immunol. 173(10), 6098-6108 (2004).

36 Mercader M, Bodner BK, Moser MT et al. T-cell infiltration of the prostate induced by androgen withdrawal in patients with prostate cancer. Proc. Natl Acad. Sci. USA 98(25), 14565-14570 (2001).

37 Tollefson M, Karnes RJ, Thompson RH et al. 668 a randomized Phase II study of ipilimumab with androgen ablation compared with androgen ablation alone in patients with advanced prostate cancer. J. Urol. 183(4), e261 (2010).

38 Spratt DE, Zhao SG, Chang SL et al. Identification and validation of intrinsic subtypes of prostate cancer. Int. J. Radiat. Oncol. Biol. Phys. 96(2), S3-S4 (2016). 
